\title{
Significance of dynamic evolution of TNF- $\alpha$, IL-6 and intestinal fatty acid-binding protein levels in neonatal necrotizing enterocolitis
}

\author{
ZHAOHUI LI and LEI SHENG \\ Department of Pediatrics, Jining First People's Hospital, \\ Jining, Shangdong 272000, P.R. China
}

Received July 19, 2017; Accepted November 1, 2017

DOI: $10.3892 /$ etm.2017.5532

\begin{abstract}
To study the significance of dynamic evolution of serum tumor necrosis factor- $\alpha$ (TNF- $\alpha)$, interleukin 6 (IL-6) and intestinal fatty acid-binding protein (I-FABP) levels in neonatal necrotizing enterocolitis (NEC). A total of 45 NEC child patients, 45 non-NEC child patients and 45 healthy newborns were enrolled. After the day age, weight, gestational week and delivery mode were matched, the serum TNF- $\alpha$, IL-6 and I-FABP levels at 6, 24 and $72 \mathrm{~h}$ after admission were measured via ELISA method, and their correlations with prognosis were analyzed. The levels of serum TNF- $\alpha$ and IL- 6 in NEC and non-NEC group reached the peak at $24 \mathrm{~h}$ and fell at $72 \mathrm{~h}$; there were no differences in each time point between the two groups $(\mathrm{P}>0.05)$, but the levels of serum TNF- $\alpha$ and IL-6 were higher than those in the control group $(\mathrm{P}<0.05)$. The level of serum I-FABP in NEC and non-NEC group reached the peak at $6 \mathrm{~h}$, and it fell at $72 \mathrm{~h}$ in NEC group and $24 \mathrm{~h}$ in non-NEC group; the level of I-FABP in each time point in NEC was significantly higher than that in non-NEC group, and the level was the lowest in healthy group; the differences were statistically significant $(\mathrm{P}<0.05)$. There were 40 cases of survival and 5 cases of death $(11.1 \%)$ in NEC group, while there were 43 cases of survival and 2 cases of death $(4.4 \%)$ in non-NEC group. There were no differences in serum TNF- $\alpha$ and IL- 6 levels at different times between surviving child patients and dead child patients in NEC group $(\mathrm{P}>0.05)$, but the levels of serum I-FABP in surviving child patients at $6 \mathrm{~h}$ and $24 \mathrm{~h}$ were significantly lower than those in dead child patients $(\mathrm{P}<0.05)$, and there was no difference at $72 \mathrm{~h}(\mathrm{P}>0.05)$.
\end{abstract}

Correspondence to: Dr Lei Sheng, Department of Pediatrics, Jining First People's Hospital, 6 Health Road, Jining, Shangdong 272000, P.R. China

E-mail: shenglei0877@163.com

Key words: TNF- $\alpha$, IL-6, intestinal fatty acid-binding protein, neonatal necrotizing enterocolitis
There were no differences in serum TNF- $\alpha$, IL-6 and I-FABP levels at different times between surviving and dead child patients in non-NEC group $(\mathrm{P}>0.05)$. Serum I-FABP level and its dynamic evolution may be important indexes of early diagnosis and prognosis evaluation of NEC.

\section{Introduction}

Neonatal necrotizing enterocolitis (NEC) is a kind of severe disease with high morbidity and mortality in neonatal period, NEC in China accounts for about 4\%o in newborns, and stage-III mortality of NEC is as high as $75-80 \%$ (1). The early manifestations are mostly atypical, mainly the general symptoms, such as abdominal distension and emesis and other feeding intolerance, dyspnea, drowsiness and body temperature fluctuation, which usually occur in premature infants with peak period of disease at 2 weeks after birth, or 2 months for low-weight child patients. After that, patients will suffer from the bloody stools, respiratory failure, shock, disseminated intravascular coagulation and even death (2). Clinical diagnosis is often made based on symptoms and signs, abdominal X-ray, abdominal ultrasound, peripheral white blood cells and differential count (3). With the development of neonatal intensive care unit (NICU) technique, the early detection of biochemical markers with high sensitivity and specificity in blood or urine has an important application value in the diagnosis and evaluation of disease. The intestinal fatty acid-binding protein (I-FABP) is a kind of protein secreted by the small intestinal simple columnar epithelial cells, which has good organ specificity. Under the ischemic injury of intestinal mucosal, abundant I-FABP can be released into the blood, and filtered by the glomeruli because it is the water-soluble protein with small molecular weight; therefore, the detection of blood and urine can reflect the intestinal ischemic injury $(4,5)$. In addition, the inflammatory response also plays an important role in the occurrence and development of NEC. A number of studies have shown that $(6,7)$ the serum tumor necrosis factor- $\alpha$ (TNF- $\alpha$ ) and interleukin 6 (IL-6) levels have a good correlation with the early diagnosis and prognosis evaluation of disease. Based on this, the study further evaluated the significance of dynamic evolution of serum TNF- $\alpha$, IL- 6 and I-FABP levels in NEC. 


\section{Materials and methods}

Object data. A total of 45 NEC child patients admitted to Jining First People's Hospital from January to December 2016 were continuously selected, 45 non-NEC children and 45 healthy newborns in the same period were selected. NEC met the bell diagnosis and classification criteria (8). In NEC group, there were 22 boys and 23 girls; aged 10-23 days (15.6 \pm 7.2 days on average); the gestational age was 32-38 weeks (34.8 2.3 weeks on average); the birth weight was $1.8-3.1 \mathrm{~kg}(2.5 \pm 0.8 \mathrm{~kg}$ on average); and there were 20 cases of cesarean delivery; 25 of vaginal delivery; 30 of premature delivery; 15 of full-term delivery; 20 of stage-I NEC; 14 of stage-II NEC; and 11 of stage-III NEC. In non-NEC group, there were 25 boys and 20 girls; aged 8-27 days (17.3 \pm 7.8 days on average); the gestational age was 33-39 weeks (35.5 \pm 2.7 weeks on average); the birth weight was $1.7-3.5 \mathrm{~kg}(2.6 \pm 0.7 \mathrm{~kg}$ on average); and there were 22 cases of cesarean delivery; 23 of vaginal delivery; 26 of premature delivery; 19 of full-term delivery; 23 of septicemia; 7 of congenital megacolon; and 15 of dysentery. In healthy newborn group, there were 24 boys and 21 girls; aged 5-28 days (17.1 \pm 7.4 days on average); the gestational age was $35-39$ weeks (37.2 \pm 2.2 weeks on average); the birth weight was $1.9-3.3 \mathrm{~kg}(2.5 \pm 0.8 \mathrm{~kg}$ on average); and there were 17 cases of cesarean delivery; 28 of vaginal delivery; 20 of premature delivery; and 25 of full-term delivery. The baseline data of children among groups were comparable. Children with congenital immune deficiency, genetic metabolic diseases, autoimmune diseases and severe infection, were excluded, and the informed consent was written and signed from the guardians of the children. The study was approved by the Ethics Committee of Jining First People's Hospital.

Research methods. The levels of serum TNF- $\alpha$, IL- 6 and I-FABP at 6, 24 and $72 \mathrm{~h}$ after admission were measured via ELISA method. A total of $5 \mathrm{ml}$ peripheral venous blood was collected, placed overnight at $4^{\circ} \mathrm{C}$ and centrifuged for $20 \mathrm{~min}$ at 2,000 $\mathrm{x} \mathrm{g}$. The supernatant was taken for detection. The kit was purchased from Sigma (Merck KGaA, Darmstadt, Germany) and the microplate reader was purchased from Bio-Rad Laboratories, Inc. (Hercules, CA, USA). The results were corrected by standard curve and detected 3 times to take the average.

Fasting, gastrointestinal decompression, intestinal probiotics, nutritional support and other conservative treatment were adopted in the treatment of NEC. If the condition of illness was not relieved, patients might suffer from the intestinal perforation or intestinal necrosis, so the surgical resection could be performed. The correlation between the above indexes and clinical prognosis was analyzed.

Statistical analysis. SPSS 20.0 (IBM Corp., Armonk, NY, USA) software was used for statistical analysis. Measurement data were presented as mean \pm standard deviation, independent sample t-test was used for comparison between two groups, one-way ANOVA was used for comparison among groups, and least significant difference t-test was used for pairwise comparison; repeated measurement ANOVA

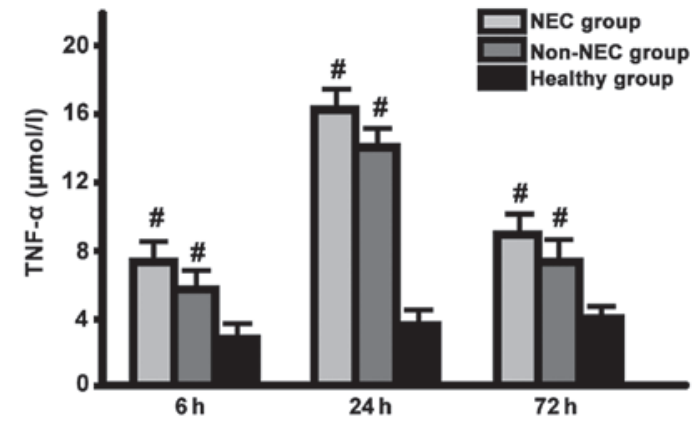

Figure 1. Detection of serum TNF- $\alpha$ levels in each group at different time points via ELISA method ( ${ }^{*}$ comparison among NEC, non-NEC and healthy group in the same time, $\mathrm{P}<0.05$; the serum TNF- $\alpha$ level in NEC and non-NEC group reached the peak at $24 \mathrm{~h}$ and fell at $72 \mathrm{~h}$; there was no difference in each time point between the two groups, but the serum TNF- $\alpha$ level was higher than that in healthy group). TNF- $\alpha$, tumor necrosis factor- $\alpha$; NEC, neonatal necrotizing enterocolitis.

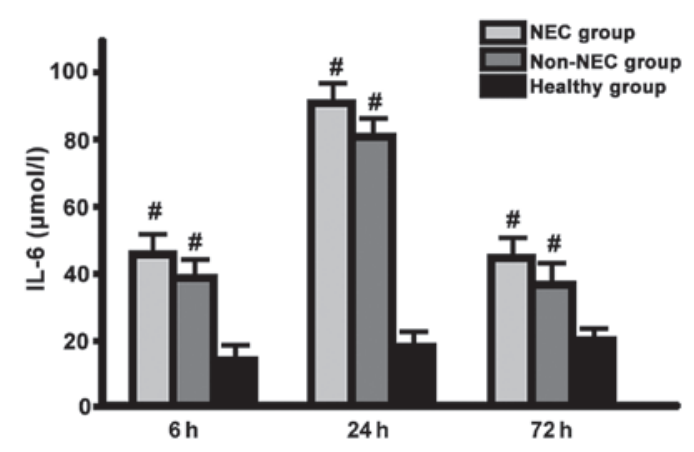

Figure 2. Detection of serum IL-6 levels in each group at different time points via ELISA method ( ${ }^{*}$ comparison among NEC group, non-NEC group and healthy group in the same time, $\mathrm{P}<0.05$; the serum IL-6 level in NEC group and non-NEC group reached the peak at $24 \mathrm{~h}$ and fell at $72 \mathrm{~h}$; there was no difference in each time point between the two groups, but the serum IL-6 level was higher than that in healthy group). Interleukin 6, IL-6; NEC, neonatal necrotizing enterocolitis.

was used for comparison of data in different time points. Enumeration data were presented as case or percentage (\%), and Chi-square test was used for comparison among groups; $\mathrm{P}<0.05$ was considered to indicate a statistically significant difference.

\section{Results}

Dynamic evolution of serum TNF- $\alpha$ level. The serum TNF- $\alpha$ level in NEC and non-NEC group reached the peak at $24 \mathrm{~h}$ and fell at $72 \mathrm{~h}$; there was no difference in each time point between the two groups $(P>0.05)$, but the serum TNF- $\alpha$ level was higher than that in healthy group $(\mathrm{P}<0.05)$ (Fig. 1).

Dynamic evolution of serum IL-6 level. The serum IL-6 level in NEC and non-NEC group reached the peak at $24 \mathrm{~h}$ and fell at $72 \mathrm{~h}$; there was no difference in each time point between the two groups $(\mathrm{P}>0.05)$, but the serum IL-6 level was higher than that in healthy group $(\mathrm{P}<0.05)$ (Fig. 2).

Dynamic evolution of serum I-FABP level. The level of serum I-FABP in NEC and non-NEC group reached the peak at $6 \mathrm{~h}$, 


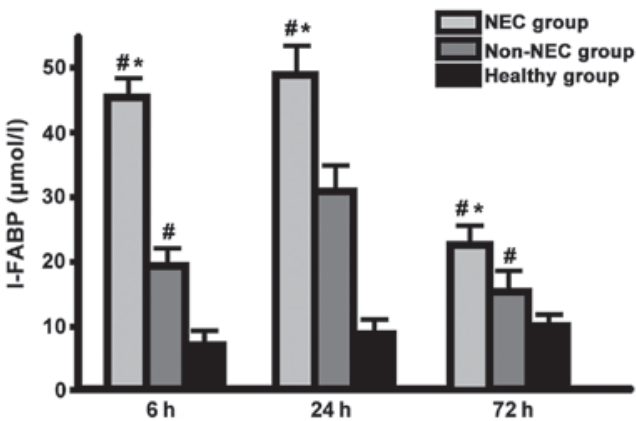

Figure 3. Detection of serum I-FABP levels in each group at different time points via ELISA method ( ${ }^{*}$ comparison among NEC group, non-NEC group and healthy group in the same time, $\mathrm{P}<0.05 ;{ }^{*}$ comparison between NEC and non-NEC group, $\mathrm{P}<0.05$; the level of serum I-FABP in NEC and non-NEC group reached the peak at $6 \mathrm{~h}$, and it fell at $72 \mathrm{~h}$ in NEC group and $24 \mathrm{~h}$ in non-NEC group; the level of I-FABP in each time point in NEC group was significantly higher than that in non-NEC group, and the level was the lowest in healthy group; the differences were statistically significant). I-FABP, intestinal fatty acid-binding protein; NEC, neonatal necrotizing enterocolitis.

and it fell at $72 \mathrm{~h}$ in NEC group and $24 \mathrm{~h}$ in non-NEC group; the level of I-FABP in each time point in NEC group was significantly higher than that in non-NEC group, and the level was the lowest in healthy group; the differences were statistically significant $(\mathrm{P}<0.05)$ (Fig. 3).

Clinical prognosis. There were 40 cases of survival and 5 cases of death $(11.1 \%)$ in NEC group, while there were 43 cases of survival and 2 cases of death $(4.4 \%)$ in non-NEC group. There were no differences in serum TNF- $\alpha$ and IL-6 levels in different time between surviving child patients and dead child patients in NEC group ( $P>0.05)$, but the levels of serum I-FABP in surviving child patients at 6 and $24 \mathrm{~h}$ were significantly lower than those in dead child patients $(\mathrm{P}<0.05)$, and there was no difference at $72 \mathrm{~h}(\mathrm{P}>0.05)$. There were no differences in serum TNF- $\alpha$, IL- 6 and I-FABP levels in different time between surviving and dead child patients in non-NEC group $(\mathrm{P}>0.05)$ (Tables I and II).

\section{Discussion}

Previous studies only simply analyzed the diagnostic value of one kind of cytokine or I-FABP level in NEC, but this study compared and analyzed the diagnostic values of TNF- $\alpha$, IL- 6 and I-FABP levels and their dynamic evolution processes in the early diagnosis and clinical prognosis of NEC. The results showed that the levels of serum TNF- $\alpha$ and IL-6 in NEC and non-NEC group reached the peak at $24 \mathrm{~h}$ and fell at $72 \mathrm{~h}$; there were no differences in each time point between the two groups, but the levels of serum TNF- $\alpha$ and IL- 6 were higher than those in healthy group, suggesting that the inflammatory response represented by TNF- $\alpha$ and IL- 6 plays an important role in NEC and many other severe neonatal diseases. TNF- $\alpha$ is an important nuclear transcriptional regulation factor in the body, which is transduced into the nucleus under the induction of a variety of stressors, such as infection and diarrhea, mediates multiple signaling transduction pathways, such as $\mathrm{NF}-\kappa \mathrm{B}, \mathrm{PI} 3 \mathrm{~K}$, Fas/FasL and MAPK, regulates a variety of genes, such as ILs, $\gamma$-IFN and VEGF, and participates in inflammatory response, oxidative stress, immune response,
Table I. Serum TNF- $\alpha$, IL-6 and I-FABP levels in different time between surviving child and dead child patients in NEC group $(\mu \mathrm{mol} / 1)$.

\begin{tabular}{ccccc}
\hline $\begin{array}{l}\text { Serum } \\
\text { levels }\end{array}$ & $\begin{array}{c}\text { Surviving child } \\
\text { patient }(\mathrm{n}=40)\end{array}$ & $\begin{array}{c}\text { Dead child } \\
\text { patients }(\mathrm{n}=5)\end{array}$ & t-test & P-value \\
\hline TNF- $\alpha$ & & & & \\
$6 \mathrm{~h}$ & $7.4 \pm 1.2$ & $7.7 \pm 1.5$ & 0.265 & 0.852 \\
$24 \mathrm{~h}$ & $15.9 \pm 2.5$ & $16.6 \pm 2.8$ & 0.452 & 0.659 \\
$72 \mathrm{~h}$ & $8.9 \pm 1.5$ & $9.3 \pm 1.7$ & 0.323 & 0.782 \\
$\mathrm{IL}-6$ & & & & \\
$6 \mathrm{~h}$ & $43.6 \pm 3.8$ & $46.5 \pm 4.4$ & 0.352 & 0.796 \\
$24 \mathrm{~h}$ & $95.7 \pm 6.6$ & $98.2 \pm 6.8$ & 0.421 & 0.765 \\
$72 \mathrm{~h}$ & $45.2 \pm 4.6$ & $48.7 \pm 4.5$ & 0.339 & 0.798 \\
$\mathrm{I}-\mathrm{FABP}$ & & & & \\
$6 \mathrm{~h}$ & $40.2 \pm 5.6$ & $49.8 \pm 7.8$ & 4.629 & 0.038 \\
$24 \mathrm{~h}$ & $44.5 \pm 8.2$ & $56.9 \pm 12.2$ & 5.352 & 0.027 \\
$72 \mathrm{~h}$ & $21.2 \pm 4.6$ & $23.5 \pm 4.8$ & 0.295 & 0.823 \\
\hline
\end{tabular}

TNF- $\alpha$, tumor necrosis factor- $\alpha$; interleukin 6 , IL-6; I-FABP, intestinal fatty acid-binding protein; NEC, neonatal necrotizing enterocolitis.

Table II. Serum TNF- $\alpha$, IL-6 and I-FABP levels at different times between surviving and dead child patients in non-NEC group $(\mu \mathrm{mol} / \mathrm{l})$.

\begin{tabular}{ccccc}
\hline $\begin{array}{l}\text { Serum } \\
\text { Levels }\end{array}$ & $\begin{array}{c}\text { Surviving child } \\
\text { patient }(\mathrm{n}=43)\end{array}$ & $\begin{array}{c}\text { Dead child } \\
\text { patients }(\mathrm{n}=2)\end{array}$ & t-test & P-value \\
\hline TNF- $\alpha$ & & & & \\
$6 \mathrm{~h}$ & $7.2 \pm 1.5$ & $7.3 \pm 1.7$ & 0.195 & 0.932 \\
$24 \mathrm{~h}$ & $14.8 \pm 4.6$ & $15.2 \pm 4.8$ & 0.325 & 0.768 \\
$72 \mathrm{~h}$ & $7.6 \pm 2.2$ & $7.9 \pm 2.5$ & 0.202 & 0.865 \\
IL-6 & & & & \\
$6 \mathrm{~h}$ & $38.6 \pm 4.5$ & $40.2 \pm 4.8$ & 0.326 & 0.768 \\
$24 \mathrm{~h}$ & $81.2 \pm 6.7$ & $82.5 \pm 6.9$ & 0.425 & 0.772 \\
$72 \mathrm{~h}$ & $36.9 \pm 5.9$ & $35.7 \pm 5.8$ & 0.317 & 0.854 \\
I-FABP & & & & \\
$6 \mathrm{~h}$ & $18.7 \pm 3.5$ & $19.5 \pm 3.7$ & 0.121 & 0.968 \\
$24 \mathrm{~h}$ & $30.2 \pm 5.4$ & $32.3 \pm 5.6$ & 0.232 & 0.854 \\
$72 \mathrm{~h}$ & $15.5 \pm 3.9$ & $14.8 \pm 3.6$ & 0.254 & 0.903 \\
\hline
\end{tabular}

TNF- $\alpha$, tumor necrosis factor- $\alpha$; interleukin 6 , IL-6; I-FABP, intestinal fatty acid-binding protein; NEC, neonatal necrotizing enterocolitis.

embryonic development, cell proliferation, differentiation and apoptosis $(9,10)$. IL-6 is an important pro-inflammatory mediator of the body and a pro-inflammatory factor of many other inflammatory cells and inflammatory media, which is one of the key factors leading to the inflammatory cascade and balance disorder $(11,12)$.

The level of serum I-FABP in NEC and non-NEC group reached the peak at $6 \mathrm{~h}$, and it fell at $72 \mathrm{~h}$ in NEC group and $24 \mathrm{~h}$ in non-NEC group; the level of I-FABP in each time point in NEC group was significantly higher than that in 
non-NEC group, and the level was the lowest in healthy group; the differences were statistically significant, suggesting that I-FABP level can be rapidly increased in the early stage of NEC, its peak is higher than that in other intestinal diseases, and the duration is also longer. NEC is characterized by ischemic necrosis of gastrointestinal tract and intestinal perforation. The pathogenesis involves intestinal hypoxia-ischemia, immature intestinal development, microbial reproduction and high immune response of intestinal mucosa $(13,14)$. I-FABP is mainly distributed in villus cells in intestinal mucosa, which can be quickly released when intestinal ischemic injury occurs, enters the blood circulation through the cell membranes, capillaries, lymphatic capillaries and portal veins, and is finally discharged with urine from the body (15). And I-FABP in blood and urine can be stably maintained at room temperature for $24 \mathrm{~h}$ (16). Schurink et al (4) studied and pointed out that the I-FABP level changed with the development of NEC symptoms, reached the peak within $24 \mathrm{~h}$ and then fell; and the duration of peak of spontaneous intestinal perforation was prolonged. I-FABP level is related to the intestinal necrosis, and the higher the I-FABP level is, the worse the clinical prognosis will be (17).

The subgroup analysis in this study showed that there were no differences in serum TNF- $\alpha$ and IL-6 levels at different times between surviving and dead child patients in NEC group, but the levels of serum I-FABP in surviving child patients at 6 and $24 \mathrm{~h}$ were significantly lower than those in dead child patients, and there was no difference at $72 \mathrm{~h}$. There were no differences in serum TNF- $\alpha$, IL- 6 and I-FABP levels at different times between surviving and dead child patients in non-NEC group. Therefore, we believe that the increased serum I-FABP level can be used as the sensitive and specific index of NEC, the early diagnosis can improve the recognition rate of disease, and its dynamic evolution may be an important index for early diagnosis and prognosis evaluation of NEC. The shortcoming of this study is the smaller sample size, and the results need to be verified by larger-sample sizes in clinical randomized controlled trials.

\section{References}

1. Wang L, Li Y and Liu J: Diagnostic value and disease evaluation significance of abdominal ultrasound inspection for neonatal necrotizing enterocolitis. Pak J Med Sci 32: 1251-1256, 2016.

2. Yu Y, Shiou S-R, Guo Y, Lu L, Westerhoff M, Sun J, Petrof EO and Claud EC: Erythropoietin protects epithelial cells from excessive autophagy and apoptosis in experimental neonatal necrotizing enterocolitis. PLoS One 8: e69620, 2013.
3. Parikh M, Samujh R, Kanojia RP and Rao KL: Decision-making in surgical neonatal necrotizing enterocolitis. J Indian Assoc Pediatr Surg 14: 102-107, 2009.

4. Schurink M, Kooi EMW, Hulzebos CV, Kox RG, Groen H, Heineman E, Bos AF and Hulscher JBF: Intestinal fatty acid-binding protein as a diagnostic marker for complicated and uncomplicated necrotizing enterocolitis: A prospective cohort study. PLoS One 10: e0121336, 2015.

5. Coufal S, Kokesova A, Tlaskalova-Hogenova H, Snajdauf J, Rygl $M$ and Kverka M: Urinary intestinal fatty acid-binding protein can distinguish necrotizing enterocolitis from sepsis in early stage of the disease. J Immunol Res 2016: 5727312, 2016.

6. Claud EC: Neonatal necrotizing enterocolitis -inflammation and intestinal immaturity. Antiinflamm Antiallergy Agents Med Chem 8: 248-259, 2009

7. McElroy SJ, Prince LS, Weitkamp J-H, Reese J, Slaughter JC and Polk DB: Tumor necrosis factor receptor 1-dependent depletion of mucus in immature small intestine: A potential role in neonatal necrotizing enterocolitis. Am J Physiol Gastrointest Liver Physiol 301: G656-G666, 2011.

8. Yang Q, Zheng F-P, Zhan Y-S, Tao J, Tan S-W, Liu H-L and Wu B: Tumor necrosis factor- $\alpha$ mediates JNK activation response to intestinal ischemia-reperfusion injury. World J Gastroenterol 19: 4925-4934, 2013

9. Shiou S-R, Yu Y, Guo Y, Westerhoff M, Lu L, Petrof EO, Sun J and Claud EC: Oral administration of transforming growth factor- $\beta 1$ (TGF- $\beta 1)$ protects the immature gut from injury via Smad protein-dependent suppression of epithelial nuclear factor $\kappa \mathrm{B}(\mathrm{NF}-\kappa \mathrm{B})$ signaling and proinflammatory cytokine production. J Biol Chem 288: 34757-34766, 2013.

10. Sonnier DI, Bailey SR, Schuster RM, Lentsch AB and Pritts TA: TNF- $\alpha$ induces vectorial secretion of IL-8 in Caco-2 cells. J Gastrointest Surg 14: 1592-1599, 2010.

11. Niño DF, Sodhi CP and Hackam DJ: Necrotizing enterocolitis: New insights into pathogenesis and mechanisms. Nat Rev Gastroenterol Hepatol 13: 590-600, 2016.

12. Cahill CM, Zhu W, Oziolor E, Yang Y-J, Tam B, Rajanala S, Rogers JT and Walker WA: Differential expression of the activator protein 1 transcription factor regulates Interleukin-1ß induction of Interleukin 6 in the developing enterocyte. PLoS One 11: e0145184, 2016.

13. Cheng S, Yu J, Zhou M, Tu Y and Lu Q: Serologic intestinal-fatty acid binding protein in necrotizing enterocolitis diagnosis: A meta-analysis. BioMed Res Int 2015: 156704, 2015.

14. Simões AL, Figueira RL, Gonçalves FL, Mitidiero LF, Silva OC, Peiró JL and Sbragia L: Temporal profile of intestinal tissue expression of intestinal fatty acid-binding protein in a rat model of necrotizing enterocolitis. Clinics (Sao Paulo) 71: 412-419, 2016.

15. Markel TA, Engelstad H and Poindexter BB: Predicting disease severity of necrotizing enterocolitis: How to identify infants for future novel therapies. J Clin Neonatol 3: 1-9, 2014.

16. Sylvester KG, Ling XB, Liu GY, Kastenberg ZJ, Ji J, Hu Z, Peng S, Lau K, Abdullah F, Brandt ML, et al: A novel urine peptide biomarker-based algorithm for the prognosis of necrotising enterocolitis in human infants. Gut 63: 1284-1292, 2014.

17. Young C, Sharma R, Handfield M, Mai V and Neu J: Biomarkers for infants at risk for necrotizing enterocolitis: Clues to prevention? Pediatr Res 65: 91R-97R, 2009.

This work is licensed under a Creative Commons Attribution-NonCommercial-NoDerivatives 4.0 International (CC BY-NC-ND 4.0) License. 